\title{
Targeting of Synthetic Gene Delivery Systems
}

\author{
Andreas G. Schätzlein* \\ Cancer Research UK Department of Medical Oncology, Beatson Laboratories, University of Glasgow, Glasgow G61 1BD, UK
}

Received 29 June 2002; accepted 19 July 2002

\begin{abstract}
Safe, efficient, and specific delivery of therapeutic genes remains an important bottleneck for the development of gene therapy. Synthetic, nonviral systems have a unique pharmaceutical profile with potential advantages for certain applications. Targeting of the synthetic vector improves the specificity of gene medicines through a modulation of the carriers' biodistribution, thus creating a dose differential between healthy tissue and the target site. The biodistribution of current carrier systems is being influenced to a large extent by intrinsic physicochemical characteristics, such as charge and size. Consequently, such nonspecific interactions can interfere with specific targeting, for example, by ligands. Therefore, a carrier complex should ideally be inert, that is, free from intrinsic properties that would bias its distribution away from the target site. Strategies such as coating of DNA carrier complexes with hydrophilic polymers have been used to mask some of these intrinsic targeting effects and avoid nonspecific interactions. Preexisting endogenous ligand-receptor interactions have frequently been used for targeting to certain cell types or tumours. Recently exogenous ligands have been derived from microorganisms or, like antibodies or phage-derived peptides, developed de novo. In animal models, such synthetic vectors have targeted remote sites such as a tumour. Furthermore, the therapeutic proof of the concept has been demonstrated for fitting combinations of synthetic vectors and therapeutic gene.
\end{abstract}

\section{INTRODUCTION}

The efficient and specific delivery of therapeutic genes to a target site is a challenge that will need to be overcome in order to tap into the promise and potential of gene medicines [1]. Over the last decade a number of promising synthetic, nonviral systems gene delivery systems have been developed and a profile of their potential advantages and disadvantages has emerged. Synthetic vectors have advantages relating to pharmaceutical issues, safety, and ease of use but tend to be less efficient than some viral systems $[2,3]$.

One of the critical issues that determine efficacy and safety of a therapeutic approach is its specificity, which is based on the recognition and exploitation of differentials between the diseased site and healthy tissue. As these differentials exist on different levels-molecular to systemic - it is crucial that each element of a potential gene medicine is selected with a view to exploit potential differences. The basic modules of a gene medicine, namely carrier, gene, and effector protein, each contribute to the overall activity and specificity profile. Further levels of specificity may be added through the use of, for example, prodrugs, which the effector protein then acts upon.

Targeting provides a generic strategy to improve the specificity of a pharmaceutical formulation independently of the specificity of the drug or gene itself, primarily by creation of a dose differential between healthy and diseased tissue. This review will examine strategies and specific challenges relating to the targeting of synthetic gene vector systems.

\section{SYNTHETIC GENE DELIVERY VECTORS}

Viral and nonviral synthetic, nonviral systems gene delivery vectors are characterised by a profile of potential pharmaceutical advantages and disadvantages, which need to be matched to the therapeutic strategy [3]. While short-term expression of the gene, for example, with a synthetic vector, may be acceptable for immunisation, an integrating viral vector may provide a more sustained expression suitable for gene replacement therapy.

Important advantages of synthetic vector systems are their safety, lack of immunogenicity, very low frequency of integration, and relative ease of large-scale production, which makes them more akin to conventional pharmaceutical excipients. These systems are also very flexible with regards to the therapeutic nature of size of the gene, as even mammalian artificial chromosomes of 60 mega bases have been transfected successfully [4].

The potential disadvantage of lower efficiency presents the flip side of the coin. However, one needs to bear in mind that therapeutic efficacy will ultimately depend on the suitable combination of vector and gene. For a number of synthetic systems therapeutic potential has been demonstrated, for example, in tumour models in vivo [5]. The systems also allow repeat dosing which potentially greatly improves efficacy [6]. The duration of 
gene expression can be increased significantly by genetically optimising of the expression plasmid [7].

\section{Packaging}

One of the key concepts for the use of drug carriers in general is "packaging": delivery systems fulfil a number of generic functions analogous to a mail package, such as, for example, protection of content, ease of handling, and an address for delivery. The pharmaceutical properties of the package/delivery system are determined by the box/carrier and are largely independent from the content, which only becomes relevant once the package has been delivered.

In the case of the synthetic vector systems, the active principle (pharmacophore) is the plasmid DNA. While (naked) plasmid DNA could be regarded as a macromolecular drug, its vulnerability to enzymatic degradation (nucleases) in biological fluids $\left(t_{1 / 2}\right.$ plasma $=1.2 \mathrm{~min}$ [8]) makes a protective packaging mandatory for most applications.

"Packaging" systems based on synthetic, nonviral vectors fall into two main groups, water-soluble polymers and cationic lipids/vesicles. (Another group of synthetic carrier materials is based on the use of peptides [9] which usually contain a group of charged amino acids (eg, lysine) to complex DNA.)

\section{Complex formation and physicochemistry}

The principles that govern the formation of the different complexes are similar, but some physicochemical differences exist, which affect some of the system's biological properties.

The complex formation between plasmid DNA and the carrier is initially electrostatic, that is, induced by the attraction between the anionic DNA and the cationic carrier material. This attraction leads to the formation of a DNA-carrier aggregate, so called polyplex, in the case of polymers, or lipoplexes for cationic lipids, respectively.

The stability of these complexes depends on the strength of the electrostatic interaction and thus on the total charge and the charge density of the carrier molecule. Complex formation is not always easily controlled, as the process is influenced by stoichiometric as well as kinetic factors [10]. The resulting complexes are particles with a size ranging from less than $100 \mathrm{~nm}$ to over $1 \mu \mathrm{m}$.

The total charge of the carrier molecule also depends on the number of attachment points per molecule: in the case of a polymer binding to DNA it is a manyto-many relationship, that is, each molecule provides multiple interaction opportunities; the cationic lipids on the other hand will normally be monovalent leading to a many-to-one relationship. Therefore, the noncovalent self-assembly processes are central to stabilising these types of systems. For amphiphilic carrier material such as cationic lipids, an additional stabilising factor is based on the phase separation, which leads to the sandwiching of the DNA between lipid layers.
In general, the size of the complex will be greater than that of the starting material, for example, vesicles, and may in fact continue to increase in size and ultimately lead to precipitation if the formulation is not colloidally stable.

In general, colloidal stability is being achieved by working with an excess of cationic charge, that is, carrier material, to ensure complete coating of the DNA to create charged complexes that are stabilised by the electrostatic repulsion between the particles. The majority of synthetic gene delivery systems carry a positive charge and are thus prone to interact with negatively charged molecules and surfaces. (While DNA "packaging" in the overwhelming majority of systems is based on charge-charge interactions, a few systems (eg, for DNA vaccination) aim to encapsulate DNA in the aqueous core of a vesicle $[11,12]$.)

This nonspecific interaction of positively charged complexes with cell surface molecules such as glycosaminoglycans is in fact an important factor in determining overall uptake and downstream transfection efficiency $[13,14,15]$. While this nonspecific binding and uptake effect may be useful for in vitro transfection and locoregional applications, it also introduces the potential, for a number of interactions in vivo that will bias the biodistribution and may compromise the stability of the delivery systems [2].

The adsorption of serum proteins can induce a number of effects such as complex destabilisation, aggregation, or retargeting. The binding of cellular elements such as erythrocytes [16] and platelets [17] can lead to extensive aggregation and potentially acute toxicity.

\section{TARGETING}

In the context of drug and gene delivery systems, we define "targeting" as any strategy that increases specificity primarily through a modulation of the carriers' biodistribution.

Given the complexity of the biological barriers that need to be overcome for the targeting to a remote site, locoregional administration represents a very pragmatic alternative to the targeting after systemic administration.

\section{Locoregional administration}

The feasibility of locoregional administration as an approach to improve specificity depends very much on the disease and the therapeutic strategy. For situations where one aims to treat a small number of easily accessible disease sites, it will in fact often be the method of choice. Furthermore, it is not necessarily required for the gene complex itself to reach the diseased site: local production of a remotely acting effector protein may be more efficient (eg, local production of growth factors which act on a tumour or APC stimulation for immunotherapy).

In a mouse model of intrahepatic hepatocellular carcinoma, the advantages of localised application become 
evident; transgene expression and toxicity were clearly superior after intratumour application of the gene medicine compared to either administration into the tail vein or the portal vein [18].

Anatomical barriers, such as the blood-brain-barrier, are another reason for the use of locoregional administration, which separate the organ from the systemic circulation. The blood-brain barrier seals the brain off from the systemic circulation and local administration of gene medicines is a practical way of circumventing this barrier. The intrathecal administration of a lipoplex with antiapoptotic transgenes into the cerebrospinal fluid alleviated postischemic damage in a model of ischemic brain damage [19].

One of the most important parenteral administration routes is through the oral/nasal mucosa and the lung. Local delivery using the lung is the first choice for the therapy of cystic fibrosis, that is, typically aiming to replace the faulty CFTR gene to restore the faulty transport of salt in the lung epithelium. Clinical trials for cystic fibrosis using lipoplexes were among the first to establish the safety of these vectors [20] and the efficacy of repeat dosing [6] in a clinical setting.

Although locoregional administration offers many advantageous route-specific barriers, for example, sputum [21] may need to be taken into consideration and carriers optimised accordingly [22].

\section{Targeting after systemic administration}

Targeting in drug and gene delivery usually has a narrower context than suggested in the above definition and refers to approaches, which aim to achieve a differential in drug concentration between a remote target site and the body in general.

When trying to target drugs or DNA complexes to a remote site in the body, the simplest starting point is an intravenous injection; any other means of administration (eg, intramuscular or subcutaneous injection) leads to a greater complexity of the system as additional barriers will have to be overcome to gain access to the systemic circulation.

Conventionally, targeting strategies have been categorized as either "active" or "passive." We suggest a more useful way of thinking about targeting of delivery systems may be to distinguish between targeting properties intrinsic to the carrier system, which are largely based on the systems' physicochemistry, and independent, extrinsic targeting functions, for example, ligands (cf. Figure 1).

To allow specific ligand-directed distribution to a target site, a carrier/complex should ideally be inert, that is, free from inherent properties that would bias the biodistribution. (Coming back to the picture of the "package," one could say that the site of delivery should be determined only by the address label and that the nature of the box should not influence the way the parcel is being processed.)

\section{INTRINSIC TARGETING}

Because of the complexity of biological systems and the plethora of potential interactions, it could be argued that there is no such thing as an untargeted delivery system: for any set of intrinsic physicochemical carrier properties there are specific biological interactions and effects that will bias its biodistribution. In order to be able to specifically target synthetic gene delivery systems, it is therefore important to understand the bias of biodistribution inherent to the systems' physicochemistry. In the context of a suitable therapeutic strategy, the intrinsic properties of selected systems can actually facilitate an accumulation at a remote target site.

\section{Charge}

While the cationic charge of DNA complexes complicates the systemic administration of such gene medicines, it is clear that this interaction produces a distinctive pattern of biodistribution [17] which may potentially be used with an advantage to target sites of increased vascular growth such as tumours. Some cationic vesicles have been shown to have a 15-33-fold higher uptake in angiogenic endothelial cells of the tumour neovasculature than in corresponding normal endothelial cells [23].

After intravenous administration of DNA complexes, expression in the lung tends to be one to two orders of magnitude higher than in other organs. The mechanisms behind the lung targeting effect are not entirely clear [24] but have frequently been linked to carrier charge [25]. There is also evidence that the nature of the carrier materials plays a role: lung endothelial cells show signs of active transport of PEI complexes [26] with a potential involvement of an endogenous polyamine transporter [27].

While charge is an intrinsic property of most synthetic vectors, it may also be utilised as an extrinsic targeting function, for example, for the retargeting of adenovirus to the lung [28].

\section{Size}

Complex size is a potentially important property of synthetic gene delivery vectors. DNA complexes tend to form particulate systems (ie, size range of $0.05-1 \mu \mathrm{m}$ ) with the exact size depending on a number of factors such as, for example, DNA to carrier ratio, total concentrations, ionic strength of the buffer, and kinetics of mixing. The lower limit of the particles' size is not easily adjustable and is thus intrinsic to any given formulation.

For many gene therapy applications, the target cells will form a part of the parenchyma or interstitium of the organ and the access to these cells is restricted for particulate drug carriers after vascular administration. This is because macromolecules and particulate carriers can only extravasate from the vasculature at specialised sites, for example, the liver or spleen, where the endothelial lining has suitable gaps, so called fenestrae, which allow particles of around $200 \mathrm{~nm}$ or smaller to pass [29]. 


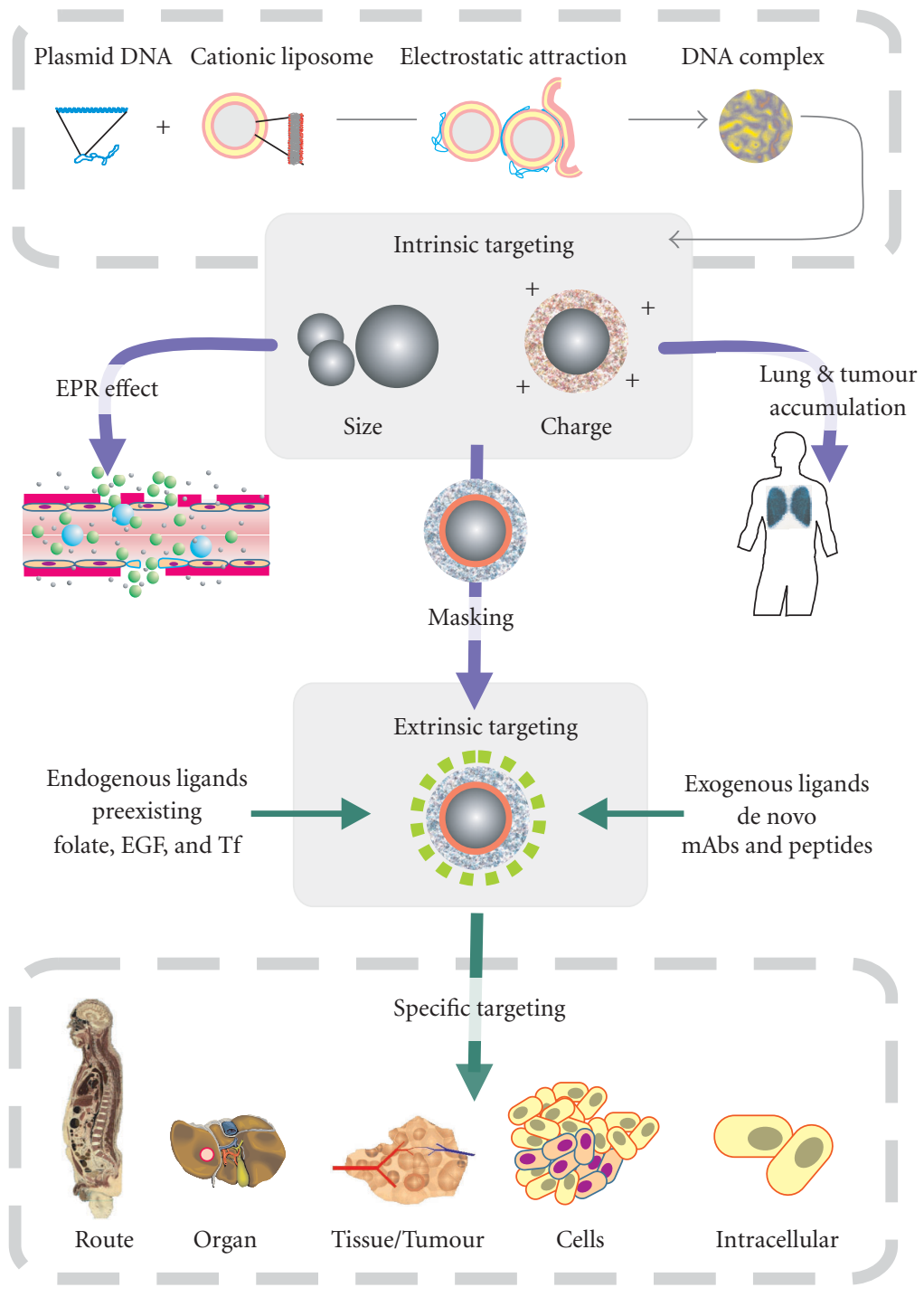

FIGURE 1. Targeting of synthetic vector systems. Schematic summary of factors important for the specific targeting of synthetic gene delivery systems. The mechanism of DNA packaging by cationic vectors, for example, vesicle, leads to the formation of condensed particles, which carry a positive charge. Particle size and charge are intrinsic physicochemical properties of these DNA complexes that, in interaction with the body, result in a modified biodistribution (eg, lung or tumour accumulation). In order to allow the use of more specific ligands, intrinsic targeting functions need to be masked, for example, by coating with hydrophilic polymers. These more inert carriers can then be directed specifically to a remote target site through the use of endogenous or exogenous ligands to allow targeting of differentials between target site and healthy tissue on various levels.

Thus, size can become a potentially important intrinsic property of synthetic gene delivery systems that will limit organ access and modulate biodistribution at the cellular level [17, 30, 31, 32].

While many studies compare the relative transfection efficiencies of formulations using "bulk reporter assays" (eg, based on organ lysates using luminescence), there is less information available about the histological distribution of the complexes and transgene expression within an organ [33]. In fact, most assays do not provide a straightforward link between quantitation and distribution of transgene expression.
The lung and liver tend to be the main organs of biodistribution after systemic administration and parenchymal expression has been reported in both of these organs. In the case of the liver, the fenestrations may play an important role in allowing gene medicines access to the parenchyma and increased hydrostatic pressure has been used to widen those gaps and thus improve delivery [34].

For the lung, it is not clear what factors are involved in overcoming the endothelial barriers but an active transcellular transport has been observed for polyethylenimine (PEI) polyplexes in the lung [26].

The bias of biodistribution introduced by complex 
size however, can also potentially be used for targeting: solid tumours growing over a size of a few millimetres recruit additional blood supply in the process of neovascularisation [35] which produces rapidly sprouting vessels with a "leaky" endothelial lining [36, 37]. Drug carriers can extravasate this "leaky" tumour vasculature and accumulate in perivascular clusters within the tumour. This "enhanced penetration and retention" (EPR) effect [38] allows the targeting of long circulating macromolecular or particulate drug carriers to tumours [39, 40, 41] and could potentially also be used with suitable synthetic gene delivery systems.

Both, charge and size of the carrier thus represent important intrinsic properties of a formulation but many other aspects of physicochemistry will potentially also influence the biodistribution. This intrinsic bias will often interfere with more specific targeting efforts. However, the example of the EPR effect-based targeting to tumours demonstrates that these effects could potentially also be used for the targeting of a suitable gene medicine.

Targeting based on the physicochemical properties of the package is not necessarily based only on the intrinsic properties: the addition of cationic charges to uncharged virus particles demonstrates the utility of charge as an extrinsic factor for the retargeting of adenovirus to the lung [28].

\section{Minimizing biodistribution bias}

Strategies to minimise bias from these intrinsic effects have been predominately focused on charge shielding and steric stabilisation.

In drug targeting, the method of using water-soluble PEG chains which create a steric barrier around the drug carrier is well established [42] and has since been applied to various gene delivery systems. PEG-phospholipid conjugates have been used to stabilise lipoplexes [43] and polyplexes. Polyplex stabilisation with PEG was achieved through the use of polylysine block [44] or comb type copolymers [45, 46], also after adsorption [47] or covalent coupling [16] to PEI polyplexes, and by covalent coupling to chitosan nanoparticles [48].

Other water-soluble materials that have helped to reduce the effects of charge-based interactions are HPMA, which has been used in conjunction with polylysine [49] or PEI polyplexes [50] and block copolymers of ethylene oxide and propylene oxide (Pluronic) [51]. Interestingly, these polymers can also be used for the retargeting of adenovirus [52].

Alternatively, the encapsulation of cationic complexes in standard liposomes can be used to reduce nonspecific interactions [53].

In addition to polymers, the coating of complexes with protein could be used to mask positive charges, although the protein itself may also introduce a bias of biodistribution. Transferrin (Tf), a popular ligand for targeting to the transferrin receptor, also can be used to cre- ate negatively charged complexes [54] or to mask the positive charge of PEI Tf polyplexes [55].

To achieve high-level transgene expression after successful minimisation of the nonspecific interactions, it is necessary to introduce an extrinsic functionality that mediates efficient targeting and cellular uptake and replaces the nonspecific binding and uptake.

\section{EXTRINSIC TARGETING}

In general, extrinsic targeting functions can be most efficiently utilised when DNA-complexes are sufficiently inert, that is, their intrinsic, nonspecific interactions do not unfavorably bias the biodistribution and interfere with the targeting.

Such vectors can in fact be considered "detargeted," as the lack of nonspecific interactions leads to a reduced cellular uptake and thus frequently makes these complexes less efficient in vitro than standard complexes. However, once such complexes have been complemented by an extrinsic targeting function they are well suited for specific, high-resolution targeting in vivo. For the majority of applications, the extrinsic targeting functionality is based on ligand-receptor interactions but other approaches such as, for example, the direction of paramagnetic complexes through the use of an external magnetic field are also being explored [56].

The binding of a "ligand" to a "receptor" (in the broadest sense) provides the basis for most specific biological interactions and provides the blueprint for ligandbased targeting strategies.

It is from this group of preexisting, "natural" ligands or receptor substrates, many of the candidates used to target synthetic gene delivery systems have initially been selected.

\section{Preexisting ligands Endogenous}

The most widely used preexisting targeting ligands are based on endogenous molecules, which are already present in the body. Preexisting ligands are often relatively easily available and the receptor and its distribution are fairly well studied.

Their use for targeting is based on the fact that a differential of expression levels exists between the target site, for example, tumour, and healthy tissue. A potential disadvantage of these ligands is the background expression of the receptor in healthy tissues and the interference of circulating endogenous ligand molecules.

The use of the vitamin folic acid as a ligand is an examples of the effective use of the natural receptor substrate for targeting. The development of targeted synthetic vectors based on folate is a natural extension of the use of this ligand for the targeting of drugs and imaging agents [57]. The receptor for the vitamin, folic acid, is overexpressed on a number of human tumors, including cancers of the ovary, kidney, uterus, testis, brain, colon, 
lung, and myelocytic blood cells [57]. In gene delivery, folic acid has been used for the targeting of liposomeencapsulated complexes [53], polylysine polyplexes [58], and polyethylenimine [59] polyplexes to receptor-positive cells.

A recent report [60] illustrates the effect of intrinsic bias on the targeting of folate lipoplexes in vivo: the lung accumulation of the cationic complexes was successfully reduced using masking with PEG polymer but targeting of these complexes with folate failed to increase the accumulation of the complexes in receptor-positive tumours, probably because other intrinsic factors were overriding the specific effects. However, the folate ligand did have a positive effect on transfection efficiency in cells that had taken up the complex.

For targeting of poly-L-lysine DNA-complexes to the liver, asialoorosomucoid [61, 62] and galactose [63, 64] have been used as ligands. While uptake in hepatocytes has been demonstrated for such systems, some of the liver targeting may be caused by the tendency of the liver, and specifically cells of the reticuloendothelial system (Kupfer cells), to "filter" out particulate drug carriers.

Since its early use for the targeting to erythroblasts [65] transferrin has become one of the most widely used ligands for targeting of synthetic vectors. Transferrin receptors are found on the surface of most proliferating cells and, in elevated numbers, on erythroblasts and on many tumours [66, 67]. Its presence on various cell types potentially limits the specificity of transferrin-targeted vectors. There have been suggestions that the effects of transferrin on gene delivery may be nonspecific and not related to targeting [68]. It has been used in conjunction with lipoplexes [69], polyplexes prepared from polylysine [70], PEI [71], or chitosan [48], and with DNA-gelatin nanoparticles and DNA-binding peptides [72].

Another important potential ligand for tumour targeting is the epidermal growth factor (EGF) [73], which has been used to target polylysine complexes [74], liposomes [75], PEI polyplexes [74], and adenovirus-derived peptides [76] to receptor-positive cells. There is currently only limited experience with the use of EGF-targeted vectors for targeting of synthetic vectors in vivo [77].

\section{Exogenous}

Many pathogenic organisms have coevolved with their hosts and developed sophisticated targeting capabilities that allow them to home on specific tissues and infect a particular cell type [78]. If this targeting functionality can be isolated from the organism's pathogenicity, such ligands would potentially be useful for targeting.

The malaria circumsporozoite (CS) protein, which coats the entire surface of sporozoites of malaria parasites and has been shown to bind specifically to the basolateral surface of hepatocytes after intravenous injection and also to target polylysine polyplexes to these cells [79]. The pa- pilloma virus capsid [80] is an example of a virus-derived targeting ligand optimised by coevolution.

\section{De novo identified ligands}

The development of targeted gene delivery vectors tends to mirror the progress in drug delivery but with a focus on the adaptation of techniques to the specific challenges of synthetic gene delivery vectors. A number of technologies for the de novo selection of potential binding ligands such as, for example, antibodies, phage display, combinatorial peptide, or nucleotide (aptamer) libraries, have recently become widely available and recombinant technology has greatly accelerated the discovery process. (Currently, phage display [81] drives the move to smaller targeting moieties such as antibody fragments $[82,83]$ and peptides $[84,85]$.) Ligands derived from these technologies have a number of advantages, in particular with respect to pharmaceutical, regulatory, and production issues, and some have already been tested in the clinic.

Antibodies to the transferrin receptor [82], the antiplatelet endothelial cell adhesion molecule PECAM [86], the polymeric immunoglobulin receptor pIgR [83], antiCD5 [87], and the ErbB-2 receptor [88] have been used for the targeting of liposomes [11,89], polylysine polyplexes [90], PEI polyplexes [86], and DNA-peptide complexes [91]. These systems in general mediated an increased uptake or expression in vivo compared to the untargeted vector.

\section{SUMMARY AND CONCLUSIONS}

Safe, efficient, and specific delivery remains a potential bottleneck for the further development of gene therapy. Each of the current systems has advantages and disadvantages and the selection of a suitable vector needs to be seen in context with the therapeutic strategy.

Targeting strategies aim to increase the specificity of a gene delivery formulation primarily through a modulation of the carriers' biodistribution, so that a dose differential is created between healthy tissue and the target site. In its simplest form, this can often be achieved by locoregional administration of the formulation, for example, intratumoural injection or inhalation for treatment of the lung. Systemic, intravenous administration of gene delivery system potentially allows targeting to (multiple) remote sites. To allow specific distribution to the target site, a carrier/complex should ideally be inert, that is, free of intrinsic properties that would bias its biodistribution.

Synthetic vector systems package the therapeutic DNA in order to protect it from degradation, deliver it to the target cells, and finally shuttle it to the nucleus to allow expression of the transgene. Current systems protect the DNA through the formation of electrostatic complexes between cationic carrier and anionic DNA. The complexes are particulate systems and tend to be positively charged. Intrinsic physicochemical characteristics of the complex such as size and charge can strongly influence the biological properties. 
The bias of biodistribution introduced by the intrinsic physicochemical properties of synthetic delivery systems frequently interferes with other more specific means of targeting such as ligands. However, when used in a controlled manner, these intrinsic properties can also provide a means of targeting in their own right, for example, the accumulation of particulate and macromolecular systems in tumours due to the enhanced penetration and retention effect.

Strategies such as coating of complexes with hydrophilic polymers have been used successfully to camouflage some of the interfering intrinsic properties of synthetic vectors. As nonspecific interactions form an important part of the cellular binding and uptake mechanism, the masked charge needs to be replaced in order to maintain efficient uptake and retain efficacy.

Specific targeting is most frequently achieved by using preexisting endogenous ligand-receptor interactions such as folate-folate receptor and transferrin-transferrin receptor. A potential drawback of endogenous ligand-receptor interactions for targeting is the background from soluble receptors, receptors in nontarget tissue, and the presence of circulating ligand. Antibodies are exogenous ligands, which avoid some of these problems. Furthermore, recent technologies for the identification of ligands (eg, phage display) have given access to a whole range of novel ligands such as antibodies, antibody fragments, and peptides.

A number of studies have recently demonstrated targeting of synthetic delivery systems (eg, using folate [60]) to remote sites such as a tumour in animal models. More importantly, tumour-targeted synthetic vectors in combination with a properly selected therapeutic gene can produce therapeutic effects [92].

The difficulty of separating intrinsic and extrinsic targeting effects represents a significant challenge for the further development of this field. The influence of intrinsic effects and nonspecific interactions becomes more difficult to predict in the complex in vivo environment. Consequently, the correlation between in vitro and in vivo efficacy of a delivery system is notoriously fragile. This means that well-controlled in vitro experiments are only of limited utility. The systematic optimisation of potentially important targeting parameters such as affinity/avidity, chemistry of conjugation, and steric situation of the coupling in vivo, however, is extremely challenging.

It is important to pursue the rapid technology transfer into the clinic with intelligent combinations of synthetic vector and therapeutic gene in order to validate the use of such carriers in vivo, create clinical safety profiles, and provide first indications how animal models correlate with clinical experience. Bearing in mind the limitations of current systems, it is however equally important to gain a deeper understanding of the complex correlation of physicochemistry and biology in order to be able to rationally design vectors that overcome systemic, cellular, and molecular barriers to genetic therapy.

\section{REFERENCES}

[1] Anderson WF. Human gene therapy. Nature. 1998; 392(Suppl 6679):25-30.

[2] Schätzlein AG. Non-viral vectors in cancer gene therapy: principles and progress. Anticancer Drugs. 2001;12(4):275-304.

[3] Verma IM, Somia N. Gene therapy-promises, problems and prospects. Nature. 1997;389(6648): 239-242.

[4] de Jong G, Telenius A, Vanderbyl S, Meitz A, Drayer J. Efficient in-vitro transfer of a $60-\mathrm{Mb}$ mammalian artificial chromosome into murine and hamster cells using cationic lipids and dendrimers. Chromosome Res. 2001;9(6):475-485.

[5] Anwer K, Meaney C, Kao G, et al. Cationic lipidbased delivery system for systemic cancer gene therapy. Cancer Gene Ther. 2000;7(8):1156-1164.

[6] Hyde SC, Southern KW, Gileadi U, et al. Repeat administration of DNA/liposomes to the nasal epithelium of patients with cystic fibrosis. Gene Ther. 2000;7(13):1156-1165.

[7] Yew NS, Zhao H, Przybylska M, et al. CpGdepleted plasmid DNA vectors with enhanced safety and long-term gene expression in vivo. Mol Ther. 2002;5(6):731-738.

[8] Houk BE, Hochhaus G, Hughes JA. Kinetic modeling of plasmid DNA degradation in rat plasma. AAPS PharmSci. 1999;1(3):E9.

[9] Sparrow JT, Edwards VV, Tung C, et al. Synthetic peptide-based DNA complexes for nonviral gene delivery. Adv Drug Deliv Rev. 1998;30(1-3):115-131.

[10] Huebner S, Battersby BJ, Grimm R, Cevc G. LipidDNA complex formation: reorganization and rupture of lipid vesicles in the presence of DNA as observed by cryoelectron microscopy. Biophys J. 1999;76(6):3158-3166.

[11] Wang CY, Huang L. Highly efficient DNA delivery mediated by $\mathrm{pH}$-sensitive immunoliposomes. Biochemistry. 1989;28(24):9508-9514.

[12] Perrie Y, Frederik PM, Gregoriadis G. Liposomemediated DNA vaccination: the effect of vesicle composition. Vaccine. 2001;19(23-24):3301-3310.

[13] Mislick KA, Baldeschwieler JD. Evidence for the role of proteoglycans in cation-mediated gene transfer. Proc Natl Acad Sci USA. 1996;93(22):12349-12354.

[14] Ruponen M, Yla-Herttuala S, Urtti A. Interactions of polymeric and liposomal gene delivery systems with extracellular glycosaminoglycans: physicochemical and transfection studies. Biochim Biophys Acta. 1999;1415(2):331-341.

[15] Miller CR, Bondurant B, McLean SD, McGovern KA, O'Brien DF. Liposome-cell interactions in vitro: effect of liposome surface charge on the binding and endocytosis of conventional and sterically stabilized liposomes. Biochemistry. 1998;37(37):12875-12883.

[16] Ogris M, Brunner S, Schuller S, Kircheis R, Wagner E. PEGylated DNA/transferrin-PEI complexes: 
reduced interaction with blood components, extended circulation in blood and potential for systemic gene delivery. Gene Ther. 1999;6(4):595-605.

[17] McLean JW, Fox EA, Baluk P, et al. Organ-specific endothelial cell uptake of cationic liposome-DNA complexes in mice. Am J Physiol. 1997;273(pt 2):H387-H404.

[18] Mohr L, Yoon SK, Eastman SJ, et al. Cationic liposome-mediated gene delivery to the liver and to hepatocellular carcinomas in mice. Hum Gene Ther. 2001;12(7):799-809.

[19] Cao YJ, Shibata T, Rainov NG. Liposome-mediated transfer of the bcl-2 gene results in neuroprotection after in vivo transient focal cerebral ischemia in an animal model. Gene Ther. 2002;9(6):415-419.

[20] Caplen NJ, Alton EWFW, Middleton PG, et al. Liposome-mediated CFTR gene transfer to the nasal epithelium of patients with cystic fibrosis. Nat Med. 1995;1(1):39-46.

[21] Sanders NN, Van Rompaey E, De Smedt SC, Demeester J. On the transport of lipoplexes through cystic fibrosis sputum. Pharm Res. 2002;19(4):451456.

[22] Sanders NN, De Smedt SC, Cheng SH, Demeester J. Pegylated GL67 lipoplexes retain their gene transfection activity after exposure to components of CF mucus. Gene Ther. 2002;9(6):363-371.

[23] Thurston G, McLean JW, Rizen M, et al. Cationic liposomes target angiogenic endothelial cells in tumors and chronic inflammation in mice. J Clin Invest. $1998 ; 101(7): 1401-1413$.

[24] Uyechi LS, Gagne L, Thurston G, Szoka FC Jr. Mechanism of lipoplex gene delivery in mouse lung: binding and internalization of fluorescent lipid and DNA components. Gene Ther. 2001;8(11):828-836.

[25] Barron LG, Gagne L, Szoka FC Jr. Lipoplex-mediated gene delivery to the lung occurs within 60 minutes of intravenous administration. Hum Gene Ther. 1999;10(10):1683-1694.

[26] Goula D, Becker N, Lemkine GF, et al. Rapid crossing of the pulmonary endothelial barrier by polyethylenimine/DNA complexes. Gene Ther. 2000;7(6):499504.

[27] Sokol PP, Longenecker KL, Kachel DL, Martin WJ 2nd. Mechanism of putrescine transport in human pulmonary artery endothelial cells. J Pharmacol Exp Ther. 1993;265(1):60-66.

[28] Ma Z, Mi Z, Wilson A, et al. Redirecting adenovirus to pulmonary endothelium by cationic liposomes. Gene Ther. 2002;9(3):176-182.

[29] Torchilin VP. Affinity liposomes in vivo: factors influencing target accumulation. J Mol Recognit. 1996;9(5-6):335-346.

[30] Griesenbach U, Chonn A, Cassady R, et al. Comparison between intratracheal and intravenous administration of liposome-DNA complexes for cystic fibrosis lung gene therapy. Gene Ther. 1998;5(2):181-188.

[31] Liu Y, Mounkes LC, Liggitt HD, et al. Factors influencing the efficiency of cationic liposomemediated intravenous gene delivery. Nat Biotechnol. 1997;15(2):167-173.

[32] Hofland HE, Nagy D, Liu JJ, et al. In vivo gene transfer by intravenous administration of stable cationic lipid/DNA complex. Pharm Res. 1997;14(6):742749.

[33] Zhu N, Liggitt D, Liu Y, Debs R. Systemic gene expression after intravenous DNA delivery into adult mice. Science. 1993;261(5118):209-211.

[34] Liu F, Song Y, Liu D. Hydrodynamics-based transfection in animals by systemic administration of plasmid DNA. Gene Ther. 1999;6(7):1258-1266.

[35] Eatock MM, Schätzlein AG, Kaye SB. Tumour vasculature as a target for anticancer therapy. Cancer Treat Rev. 2000;26(3):191-204.

[36] Jain RK. Delivery of molecular and cellular medicine to solid tumors. Adv Drug Deliv Rev. 1997;26(23):71-90.

[37] Hashizume H, Baluk P, Morikawa S, et al. Openings between defective endothelial cells explain tumor vessel leakiness. Am J Pathol. 2000;156(4):13631380.

[38] Maeda H. The tumor blood vessel as an ideal target for macromolecular anticancer agents. J Control Release. 1992;19(1-3):315-324.

[39] Yuan F, Leunig M, Huang SK, Berk DA, Papahadjopoulos D, Jain RK. Microvascular permeability and interstitial penetration of sterically stabilized (stealth) liposomes in a human tumor xenograft. Cancer Res. 1994;54(13):3352-3356.

[40] Gabizon A, Catane R, Uziely B, et al. Prolonged circulation time and enhanced accumulation in malignant exudates of doxorubicin encapsulated in polyethylene-glycol coated liposomes. Cancer Res. 1994;54(4):987-992.

[41] Vasey PA, Kaye SB, Morrison R, et al. Phase I clinical and pharmacokinetic study of PK1 [N-(2hydroxypropyl)methacrylamide copolymer doxorubicin]: first member of a new class of chemotherapeutic agents-drug-polymer conjugates. Cancer Research Campaign Phase I/II Committee. Clin Cancer Res. 1999;5(1):83-94.

[42] Winterhalter M, Frederik PM, Vallner JJ, Lasic DD. Stealth(R) liposomes: from theory to product. Adv Drug Deliv Rev. 1997;24(2-3):165-177.

[43] Hong K, Zheng W, Baker A, Papahadjopoulos D. Stabilization of cationic liposome-plasmid DNA complexes by polyamines and poly(ethylene glycol)phospholipid conjugates for efficient in vivo gene delivery. FEBS Lett. 1997;400(2):233-237.

[44] Katayose S, Kataoka K. Remarkable increase in nuclease resistance of plasmid DNA through supramolecular assembly with poly(ethylene glycol)-poly(L-lysine) block copolymer. J Pharm Sci. 1998;87(2):160-163.

[45] Choi YH, Liu F, Kim JS, Choi YK, Park JS, Kim SW. Polyethylene glycol-grafted poly-L-lysine as 
polymeric gene carrier. J Control Release. 1998;54(1): 39-48.

[46] Brown MD, Schätzlein AG, Brownlie A, et al. Preliminary characterization of novel amino acid based polymeric vesicles as gene and drug delivery agents. Bioconjug Chem. 2000;11(6):880-891.

[47] Blessing T, Kursa M, Holzhauser R, Kircheis R, Wagner E. Different strategies for formation of pegylated EGF-conjugated PEI/DNA complexes for targeted gene delivery. Bioconjug Chem. 2001;12(4):529-537.

[48] Mao HQ, Roy K, Troung-Le VL, et al. ChitosanDNA nanoparticles as gene carriers: synthesis, characterization and transfection efficiency. J Control Release. 2001;70(3):399-421.

[49] Toncheva V, Wolfert MA, Dash PR, et al. Novel vectors for gene delivery formed by self-assembly of DNA with poly(L-lysine) grafted with hydrophilic polymers. Biochim Biophys Acta. 1998;1380(3):354368.

[50] Oupicky D, Ogris M, Howard KA, Dash PR, Ulbrich $\mathrm{K}$, Seymour LW. Importance of lateral and steric stabilization of polyelectrolyte gene delivery vectors for extended systemic circulation. Mol Ther. 2002;5(4): 463-472.

[51] Ochietti B, Guerin N, Vinogradov SV, et al. Altered organ accumulation of oligonucleotides using polyethyleneimine grafted with poly(ethylene oxide) or pluronic as carriers. J Drug Target. 2002;10(2): 113-121.

[52] Fisher KD, Stallwood Y, Green NK, Ulbrich K, Mautner V, Seymour LW. Polymer-coated adenovirus permits efficient retargeting and evades neutralising antibodies. Gene Ther. 2001;8(5):341-348.

[53] Lee RJ, Huang L. Folate-targeted, anionic liposomeentrapped polylysine-condensed DNA for tumor cell-specific gene transfer. J Biol Chem. 1996;271(14): 8481-8487.

[54] Simoes S, Slepushkin V, Gaspar R, de Lima MC, Duzgunes N. Gene delivery by negatively charged ternary complexes of DNA, cationic liposomes and transferrin or fusigenic peptides. Gene Ther. 1998; 5(7):955-964.

[55] Kircheis R, Wightman L, Schreiber A, et al. Polyethylenimine/DNA complexes shielded by transferrin target gene expression to tumors after systemic application. Gene Ther. 2001;8(1):28-40.

[56] Scherer F, Anton M, Schillinger U, et al. Magnetofection: enhancing and targeting gene delivery by magnetic force in vitro and in vivo. Gene Ther. 2002;9(2):102-109.

[57] Wang S, Low PS. Folate-mediated targeting of antineoplastic drugs, imaging agents, and nucleic acids to cancer cells. J Control Release. 1998;53(1-3):3948.

[58] Mislick KA, Baldeschwieler JD, Kayyem JF, Meade TJ. Transfection of folate-polylysine DNA complexes: evidence for lysosomal delivery. Bioconjug Chem. 1995;6(5):512-515.
[59] Guo W, Lee RL. Receptor-targeted gene delivery via folate-conjugated polyethylenimine. AAPS PharmSci. 1999;1(4):E19.

[60] Hofland HE, Masson C, Iginla S, et al. Folatetargeted gene transfer in vivo. Mol Ther. 2002;5(6): 739-744.

[61] Wu CH, Wilson JM, Wu GY. Targeting genes: delivery and persistent expression of a foreign gene driven by mammalian regulatory elements in vivo. $\mathrm{J}$ Biol Chem. 1989;264(29):16985-16987.

[62] Stankovics J, Crane AM, Andrews E, Wu CH, Wu GY, Ledley FD. Overexpression of human methylmalonyl CoA mutase in mice after in vivo gene transfer with asialoglycoprotein/polylysine/DNA complexes. Hum Gene Ther. 1994;5(9):1095-1104.

[63] Hisayasu S, Miyauchi M, Akiyama K, Gotoh T, Satoh $\mathrm{S}$, Shimada $\mathrm{T}$. In vivo targeted gene transfer into liver cells mediated by a novel galactosyl-D-lysine/Dserine copolymer. Gene Ther. 1999;6(4):689-693.

[64] Hashida M, Takemura S, Nishikawa M, Takakura Y. Targeted delivery of plasmid DNA complexed with galactosylated poly(L-lysine). J Control Release. 1998;53(1-3):301-310.

[65] Stavridis JC, Deliconstantinos G, Psallidopoulos MC, Armenakas NA, Hadjiminas DJ, Hadjiminas J. Construction of transferrin-coated liposomes for in vivo transport of exogenous DNA to bone marrow erythroblasts in rabbits. Exp Cell Res. 1986; 164(2):568-572.

[66] Phylchenkov AA, Slukvin I, Kudryavets YI, et al. Expression and functional-activity of transferrin receptor in human tumor-cell of different histogenesis. Eksperimentalnaya Onkologiya. 1992;14(6):22-27.

[67] Goding J. CD71. NCBI, 1998. http://www.ncbi.nlm. nih.gov/PROW/guide/1445562251_g.htm.

[68] Simoes S, Slepushkin V, Pires P, Gaspar R, de Lima MP, Duzgunes N. Mechanisms of gene transfer mediated by lipoplexes associated with targeting ligands or $\mathrm{pH}$-sensitive peptides. Gene Ther. 1999;6(11):1798-1807.

[69] Cheng PW. Receptor ligand-facilitated gene transfer: enhancement of liposome-mediated gene transfer and expression by transferrin. Hum Gene Ther. 1996;7(3):275-282.

[70] Cotten M, Langle-Rouault F, Kirlappos H, et al. Transferrin-polycation-mediated introduction of DNA into human leukemic cells: stimulation by agents that affect the survival of transfected DNA or modulate transferrin receptor levels. Proc Natl Acad Sci USA. 1990;87(11):4033-4037.

[71] Kircheis R, Kichler A, Wallner G, et al. Coupling of cell-binding ligands to polyethylenimine for targeted gene delivery. Gene Ther. 1997;4(5):409-418.

[72] Plank C, Tang MX, Wolfe AR, Szoka FC Jr. Branched cationic peptides for gene delivery: role of type and number of cationic residues in formation and in vitro activity of DNA polyplexes. [published erratum appears in Hum Gene Ther. 1999;10(13):2272] Hum Gene Ther. 1999;10(2):319-332. 
[73] Voldborg BR, Damstrup L, Spang-Thomsen M, Poulsen HS. Epidermal growth factor receptor (EGFR) and EGFR mutations, function and possible role in clinical trials. Ann Oncol. 1997;8(12):11971206.

[74] Cristiano RJ, Roth JA. Epidermal growth factor mediated DNA delivery into lung cancer cells via the epidermal growth factor receptor. Cancer Gene Ther. 1996;3(1):4-10.

[75] Kikuchi A, Sugaya S, Ueda H, et al. Efficient gene transfer to EGF receptor overexpressing cancer cells by means of EGF-labeled cationic liposomes. Biochem Biophys Res Commun. 1996;227(3):666671.

[76] Medina-Kauwe LK, Maguire M, Kasahara N, Kedes L. Nonviral gene delivery to human breast cancer cells by targeted Ad5 penton proteins. Gene Ther. 2001;8(23):1753-1761.

[77] Liu X, Tian P, Yu Y, Yao M, Cao X, Gu J. Enhanced antitumor effect of EGF R-targeted p21WAF-1 and GM-CSF gene transfer in the established murine hepatoma by peritumoral injection. Cancer Gene Ther. 2002;9(1):100-108.

[78] Mammen M, Choi SK, Whitesides GM. Polyvalent interactions in biological systems: implications for design and use of multivalent ligands and inhibitors. Angew Chem Int Ed Engl. 1998;37(20):2754-2794.

[79] Ding ZM, Cristiano RJ, Roth JA, Takacs B, Kuo MT. Malarial circumsporozoite protein is a novel gene delivery vehicle to primary hepatocyte cultures and cultured cells. J Biol Chem. 1995;270(8):3667-3676.

[80] Muller M, Gissmann L, Cristiano RJ, et al. Papillomavirus capsid binding and uptake by cells from different tissues and species. J Virol. 1995;69(2):948954.

[81] Nilsson F, Tarli L, Viti F, Neri D. The use of phage display for the development of tumour targeting agents. Adv Drug Deliv Rev. 2000;43(2-3):165-196.

[82] Xu L, Tang WH, Huang CC, et al. Systemic p53 gene therapy of cancer with immunolipoplexes targeted by anti-transferrin receptor scFv. Mol Med. 2001;7(10):723-734.

[83] Gupta S, Eastman J, Silski C, Ferkol T, Davis PB. Single chain Fv: a ligand in receptor-mediated gene delivery. Gene Ther. 2001;8(8):586-592.

[84] Vaysse L, Guillaume C, Burgelin I, Gorry P, Ferec C, Arveiler B. Proteolipidic vectors for gene transfer to the lung. Biochem Biophys Res Commun. 2002;290(5):1489-1498.

[85] Harbottle RP, Cooper RG, Hart SL, et al. An RGD-oligolysine peptide: a prototype construct for integrin-mediated gene delivery. Hum Gene Ther. 1998;9(7):1037-1047.

[86] Li S, Tan Y, Viroonchatapan E, Pitt BR, Huang L. Targeted gene delivery to pulmonary endothelium by anti-PECAM antibody. Am J Physiol Lung Cell Mol Physiol. 2000;278(3):L504-L511.
[87] Merwin JR, Carmichael EP, Noell GS, et al. CD5mediated specific delivery of DNA to T lymphocytes: compartmentalization augmented by adenovirus. $J$ Immunol Methods. 1995;186(2):257-266.

[88] Uherek C, Fominaya J, Wels W. A modular DNA carrier protein based on the structure of diphtheria toxin mediates target cell-specific gene delivery. J Biol Chem. 1998;273(15):8835-8841.

[89] Wang CY, Huang L. pH-sensitive immunoliposomes mediate target-cell-specific delivery and controlled expression of a foreign gene in mouse. Proc Natl Acad Sci USA. 1987;84(22):7851-7855.

[90] Trubetskoy VS, Torchilin VP, Kennel SJ, Huang L. Use of N-terminal modified poly(L-lysine)-antibody conjugate as a carrier for targeted gene delivery in mouse lung endothelial cells. Bioconjug Chem. 1992;3(4):323-327.

[91] Fominaya J, Wels W. Target cell-specific DNA transfer mediated by a chimeric multidomain protein. Novel non-viral gene delivery system. J Biol Chem. 1996;271(18):10560-10568.

[92] Kircheis R, Wightman L, Kursa M, Ostermann E, Wagner E. Tumor-targeted gene delivery: an attractive strategy to use highly active effector molecules in cancer treatment. Gene Ther. 2002;9(11):731-735.

* Corresponding author.

E-mail: A.Schatzlein@beatson.gla.ac.uk Fax: +44 1413304127; Tel: +44 1413304354 

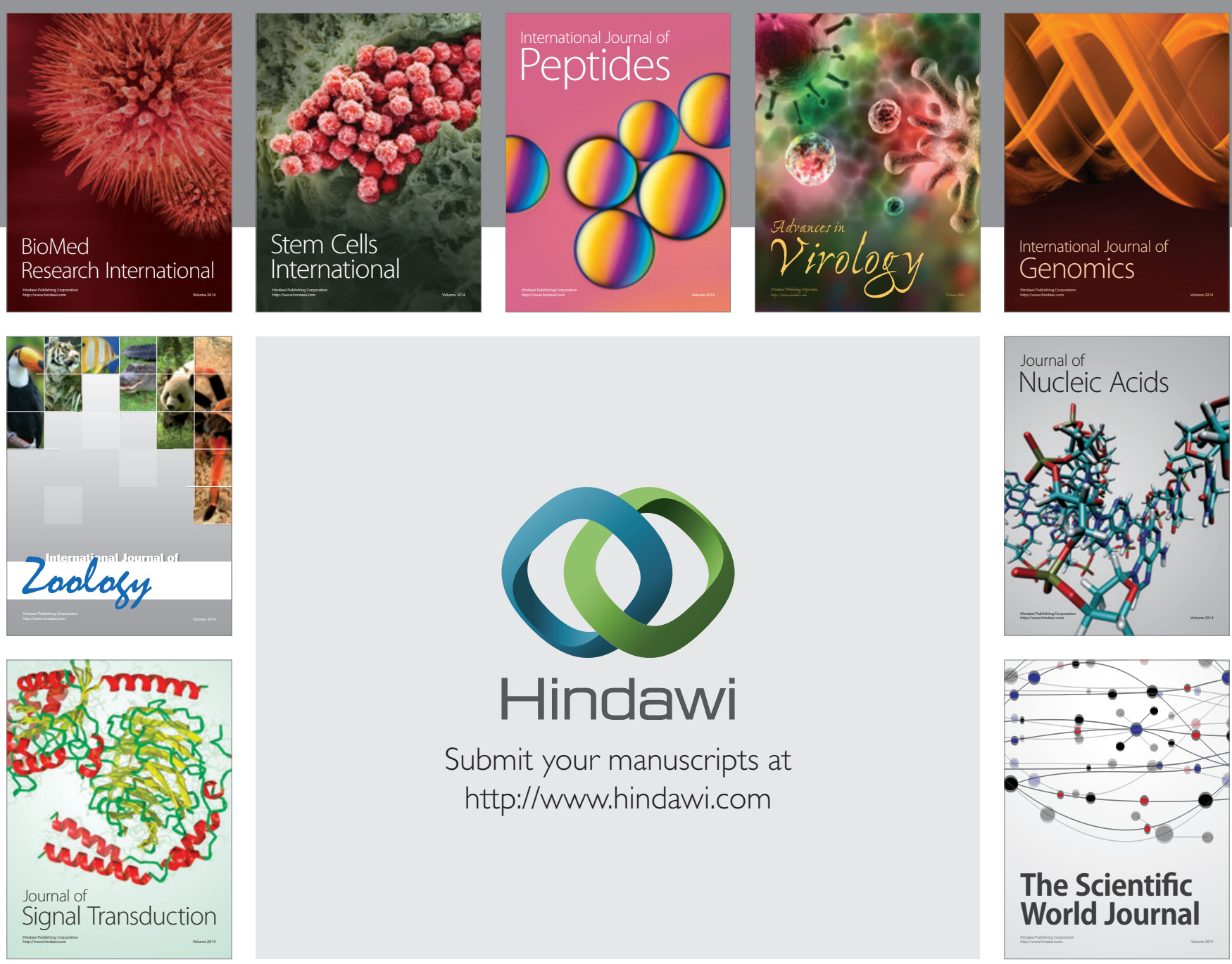

Submit your manuscripts at

http://www.hindawi.com
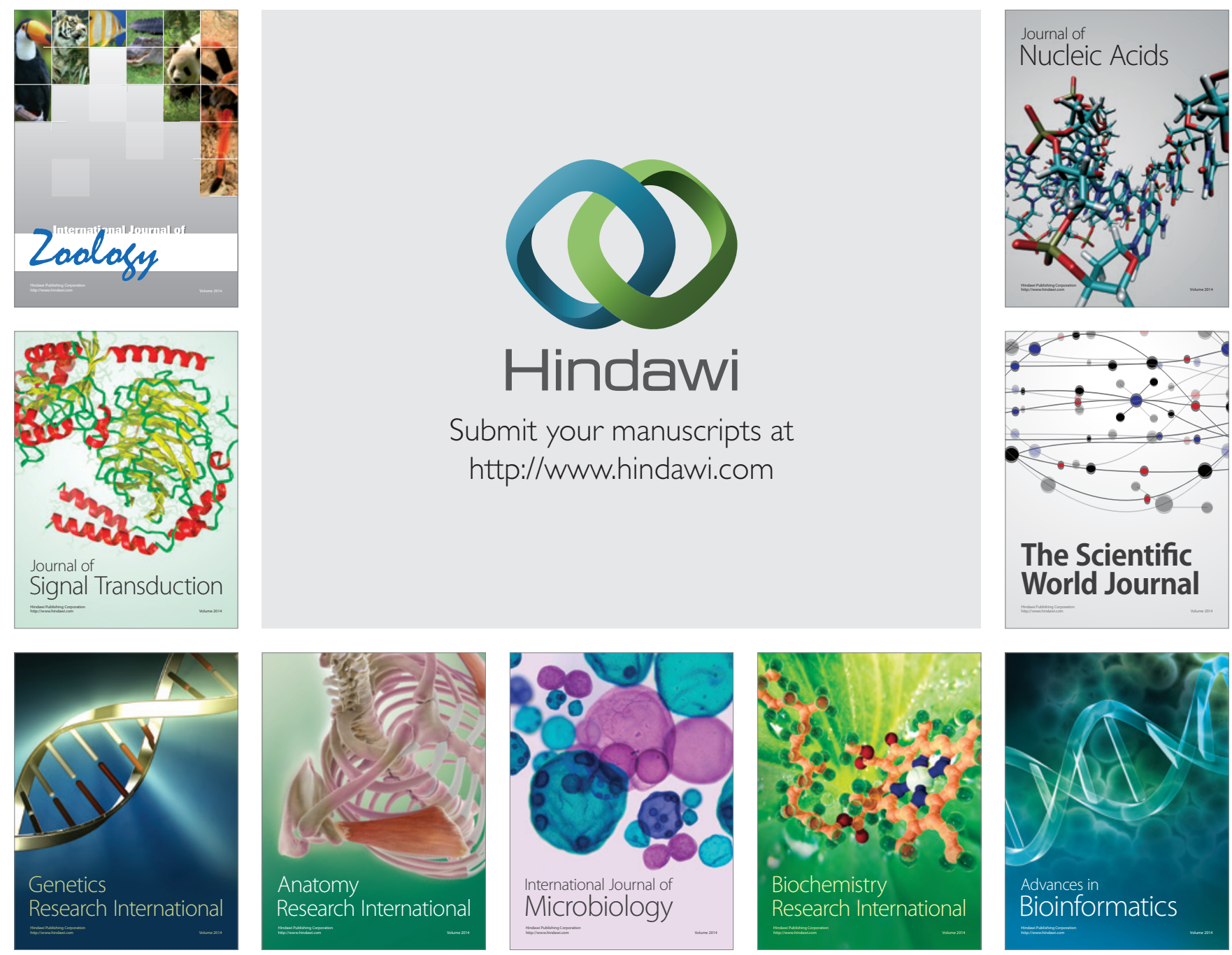

The Scientific World Journal
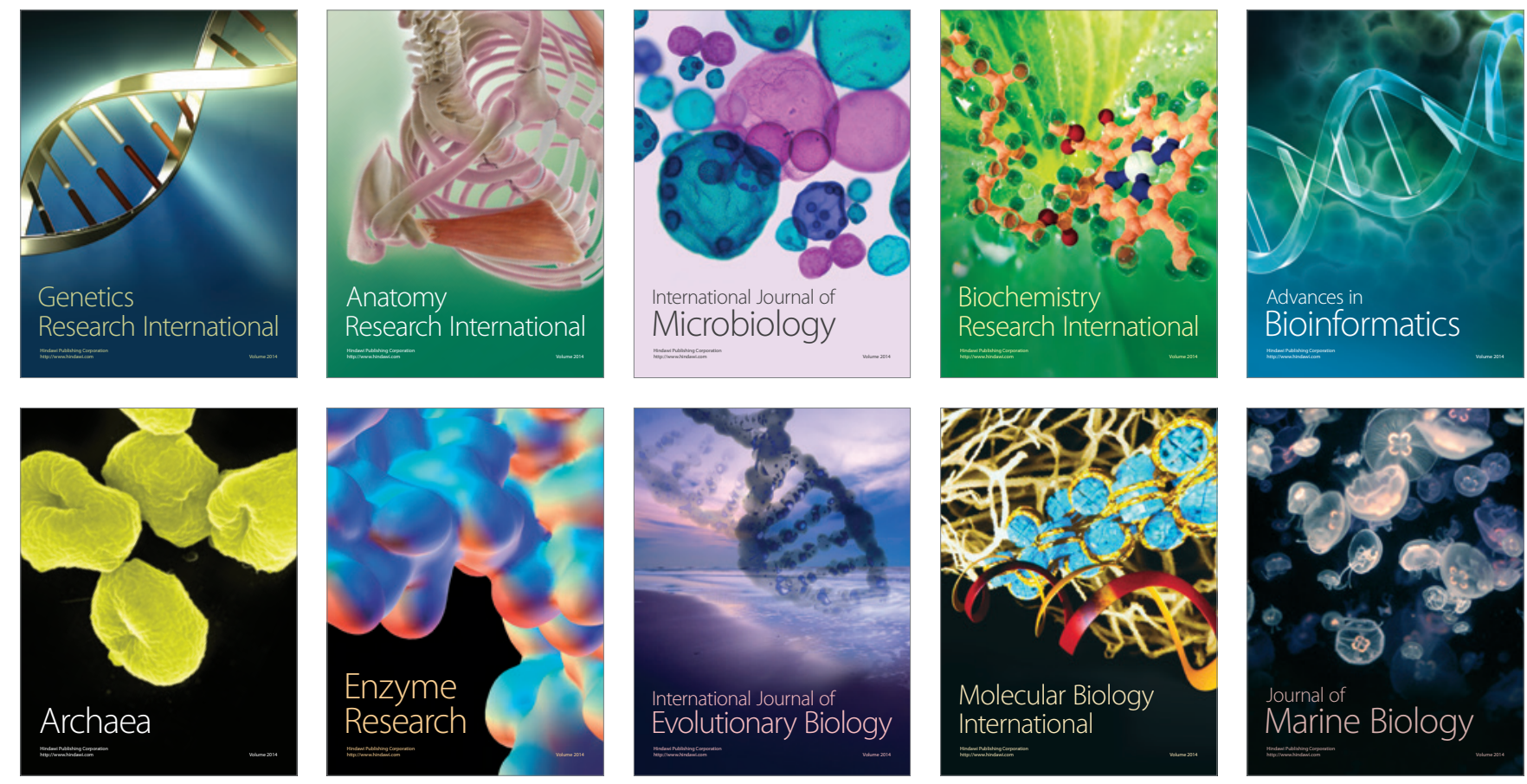